\title{
A Study on Development of Instant Kodo Dosa Mix and Evaluation of Its Nutritional Composition and Shelf Life
}

\author{
K. G. Vijayalaxmi, Jayalaxmi Baddi and Vanishri K. Umarji* \\ Department of Food Science and Nutrition, UAS, GKVK, Bengaluru, India \\ *Corresponding author
}

\section{A B S T R A C T}

\begin{tabular}{|l|}
\hline Ke y w o r d s \\
Kodo, Dosa, \\
$\begin{array}{l}\text { Free fatty acid, } \\
\text { Peroxide, Moisture, } \\
\text { Microbial load }\end{array}$ \\
\hline Article Info \\
\hline $\begin{array}{l}\text { Accepted: } \\
\text { 04 November } 2020 \\
\text { Available Online: } \\
\text { 10 December } 2020\end{array}$ \\
\hline
\end{tabular}

\section{Introduction}

Millets are nutritious crops and possesses various health benefits. Dietary fibre in millets helps to lower blood glucose level and helps to manage diabetes. Fibre binds to cholesterol and hinders its absorption, thus it helps to protect from heart diseases. Incomplete or slow fermentation of millets by micro flora in the colon, promotes normal laxation which prevents constipation, diverticulosis and diverticulitis (Mounika et al., 2017).

Minor millets are claimed to be future foods for better health and nutrition security in the recent years, they are recognized as important substitutes for major cereal crops and to cope up with the world food shortage and to meet the demands of increasing population (Shreshta and Noomhorm, 2002).

Food habits in India changed drastically due to influence of Western culture. In $21^{\text {st }}$ century ready to eat and ready to prepare foods are in demand. Instant foods help to save the time and energy.

Instant mixes are convenient food for all kind of peoples. It is easy to use and helps to reduce the effort of collecting and processing various ingredients. Qualitative and nutritious 
instant foods are the requirement of present generation. Dosa is a common breakfast food in India; it is rich in carbohydrates and protein. Main constituents of dosa are rice and black gram dhal. There are many varieties of dosa's are available at Indian kitchen. Each variation has got unique taste.

In this study attempts are made to develop value added kodo dosa mix. Muragod, P. P.et al., 2019 stated that kodo is a fantastic substitute for rice. It cooks very fast and it can be used to make a variety of dishes.

\section{Materials and Methods}

\section{Formulation and standardization of value added instant kodo millet dosa mix}

Dosa mix was prepared by using kodo millet rice flour, parboiled rice flour, fenugreek seeds powder, black gram dhal flour and salt. The following table depicts formulation of kodo dosa mix (Table 1). Procedure of kodo dosa mix preparation and kodo dosa making from the mix is depicted in fig. 1 and 2.

Sensory evaluation: Sensory evaluation of the control dosa mix and all the variations was done with the help of nine point hedonic scale by a panel of twenty one semi trained panellists.

Nutrient composition: Nutrient composition of highly accepted kodo dosa mix per $100 \mathrm{~g}$ was analyzed by following the AOAC (2000) official protocols. All samples were analyzed in triplicates. Moisture content was determined from sample weight loss after drying at $110^{\circ} \mathrm{C}$ for 4 hour. Protein content was determined by Kjeldahl method. The Soxhlet method was used for total fat determination. Crude fiber was estimated by treatment of sample first with acid and subsequently with alkali. The loss in weight was taken as the crude fibre content.
Carbohydrate and energy were analyzed by difference method (Livesey, 1995). Ash was analyzed by using muffle furnace. Calcium content of the sample was estimated by preparing mineral solution and titrating it against $0.01 \mathrm{~N}$ EDTA in the presence of alkaline condition (Heau et al., 1965). The iron content of the sample was estimated by using atomic absorption spectrophotometer and the results were expressed in mg per 100 $\mathrm{g}$ of the sample (AOAC, 1980).

Estimation of total antioxidant activity (Kang and Saltveit, 2002)

The disappearance of the DPPH (1,1Diphenyl-2-picryl hydrazyl) radical absorption at $517 \mathrm{~nm}$ by the action of antioxidants is measured spectrophotometrically in a methanolic solution until the absorbance remains constant.

\section{Estimation of total polyphenols (Sadasivam and Manickam, 1991)}

Blue colour developed by Polyphenols Folinciocalteau (FCR) reagent in alkaline condition was measured at $650 \mathrm{~nm}$.

\section{Estimation of tannins (Ranganna, 2005)}

Tannins were estimated colorimetrically based on the measurement of blue color formed by the reduction of phosphotungstomoybdic acid in alkali solution.

\section{Estimation of Phytic acid (Wheeler and Ferrel, 1971)}

The phytate was extracted with trichloroacetic acid and precipitated as ferric salt. The iron content of the precipitate was determined colorimetrically and the phytate phosphorus content calculated from value assuming a 
constant 4 Fe: 6 molecular ratios in the precipitate. Phytates were estimated as phytic and phytate phosphorous were obtained.

\section{Storage quality evaluation}

The Control and kodo dosa mixes were packed in aluminium silver pouch and plastic pouches, heat sealed and stored in cardboard boxes at ambient temperature for a period of 180 days. The storage quality of control dosa mix and kodo dosa mix were evaluated for moisture content, free fatty acid (FFA), peroxide value (PV) and sensory quality at the interval of 30 days for a period of 180 days.

\section{Microbial load}

Microbial load was analyzed by using standard protocols. Pour plate method was followed for estimation of microorganisms. For bacteria used Nutient Agar (NA), for fungi used Rose Bengal Agar (RBA), for Coliforms used Eiosine Methalene Blue Agar (EMBA). Microbial load was analyzed on initial day, $90^{\text {th }}$ day and $180^{\text {th }}$ day.

\section{Statistical analysis}

All the experiments were performed in triplicates and completely randomized design was carried out for the experimental values in order to know the significant difference (at $5 \%$ significant level).

\section{Results and Discussion}

Table 2 depicts the mean sensory scores of dosa mix for the characteristics of appearance, colour, texture, aroma, taste and overall acceptability. These variations were prepared by incorporating kodo millet flour at different levels i.e., 25, 50, 75 and 100 per cent. The scores for appearance ranged from 6.52 to 8.57, for colour 6.26 to 8.55 , texture 6.17 to 8.57 , aroma 5.80 to 8.60 , taste 5.95 to 8.60 and overall acceptability 6.00 to 8.34 . the control had the highest score for all the sensory characteristics. Among all variations, KMD $1(25 \%)$ was found to be best accepted and KMD 3(75\%) had lesser scores for all the sensory characteristics. People are familiar with only rice, black gram dhal based dosa. Hence, as the level of kodo incorporation increased, the sensory scores got reduced significantly. However, till $75 \%$ kodo flour incorporation dosa were very well acceptable between like moderately to like very much.

Vindhya and Roobhadevi (2014) reported that, dosa mix with only $20 \%$ kodo millet incorporation was best accepted due to darker colour and grainy feel of kodo millet. Roopa et al., (2017) reported that dosa mix with $40 \%$ little millet incorporation was best accepted. They also suggested that, these kinds of millet based foods can be considered as therapeutic foods for health conscious people. It was very interesting to note that in the present study, kodo millet was acceptable till 75 per cent level.

Table 3 represents the nutritional composition of $75 \%$ kodo millet incorporated dosa mix (KMD 3) in comparison with control dosa mix. Moisture (11.53\%), ash (3.49g), crude fibre $(17.33 \mathrm{~g})$, calcium $(53.83 \mathrm{mg})$ and iron $(5.62 \mathrm{~g})$ contents were significantly high in kodo millet dosa mix. Carbohydrate $(57.05 \mathrm{~g})$, protein $(12.22 \mathrm{~g})$ and energy (280.92 K.Cal) contents were significantly high in control dosa mix. Crude fibre helps to reduce the problem of lifestyle disorders such as obesity, diabetes, cancer etc., calcium helps to reduce the problem of osteoporosis and other bone related problems. Iron helps to overcome the problem of anaemia. Kodo millet based dosa mix can be used for therapeutic purpose.

Table 4 denotes the antinutritional and antioxidant composition of control and kodo dosa mix. Total antioxidants in kodo dosa mix 
was significantly high compared to control dosa mix (30.41 and $17.89 \%$ respectively). Tannins, phytic acid and polyphenols were significantly high in kodo dosa mix $(1.77 \%$, $0.85 \mathrm{~g}, 23.97 \mathrm{mg}$ respectively). Polyphenols kind of antioxidants has got antiinflammatory properties that help to reduce the problems such as CVD, neurodegenerative disorders and cancer (Pérez-Jiménez J,2010 and Singh A. 2011).

Table 5 denotes the free fatty acid values of the dosa mix from initial to $180^{\text {th }}$ day of storage. The increase in free fatty acid of control dosa mix when stored in silver pouch was from 0.28 to 0.51 , plastic pouch was from 0.29 to 0.77 which was highly significant.
In kodo dosa mix, it was observed that FFA values in silver pouch increased significantly from 0.26 to 0.75 and in plastic pouch from 0.27 to 0.88 .

FFA values were significantly high in plastic pouch compared to silver pouch both in control and kodo dosa mixes. On initial, $30^{\text {th }}$ and $60^{\text {th }}$ day of storage difference between the pouches was non - significant. However, on $90^{\text {th },} 120^{\text {th }}, 150^{\text {th }}$ and $180^{\text {th }}$ day of storage, FFA values were significantly high in plastic pouch compared to silver pouch both in control as well as in kodo dosa mix. However, in both pouches FFA values were within safe limits up to $180^{\text {th }}$ day.

Table.1 Formulation and standardization of instant kodo millet dosa mix

\begin{tabular}{|c|c|c|c|c|c|}
\hline Treatments & $\begin{array}{c}\text { Parboiled rice flour } \\
\text { (gm) }\end{array}$ & $\begin{array}{c}\text { Kodo millet } \\
\text { flour }(\mathbf{g m})\end{array}$ & $\begin{array}{c}\text { Black gram } \\
\text { dhal } \\
\mathbf{( g m}\end{array}$ & $\begin{array}{c}\text { Fenugreek } \\
\text { seeds } \\
\text { (gm) }\end{array}$ & $\begin{array}{c}\text { Salt } \\
(\mathbf{g m})\end{array}$ \\
\hline CMD & 100 & 00 & 25 & 5 & 2 \\
\hline KMD1 & 75 & 25 & 25 & 5 & 2 \\
\hline KMD2 & 50 & 50 & 25 & 5 & 2 \\
\hline KMD3 & 25 & 75 & 25 & 5 & 2 \\
\hline KMD4 & 00 & 100 & 25 & 5 & 2 \\
\hline
\end{tabular}

CMD -Control Millet Dosa, KMD(1)-Kodo Millet Dosa (25\% Kodo flour incorporation), KMD(2)-Kodo Millet Dosa (50 \% Kodo flour incorporation), KMD(3)-Kodo Millet Dosa (75\% Kodo flour incorporation), KMD(4)-Kodo Millet Dosa (100\% Kodo flour incorporation)

Table.2 Mean sensory scores of value added instant kodo millet dosa mix

\begin{tabular}{|l|c|l|l|l|c|c|}
\hline Variations & Appearance & Colour & Texture & Aroma & Taste & $\begin{array}{c}\text { Overall } \\
\text { acceptability }\end{array}$ \\
\hline CMD & $8.57 \pm 0.36$ & $8.55 \pm 0.49$ & $8.57 \pm 0.20$ & $8.60 \pm 0.26$ & $8.60 \pm 0.23$ & $8.34 \pm 0.35$ \\
\hline KMD1 & $8.34 \pm 0.34$ & $8.21 \pm 0.36$ & $8.31 \pm 0.24$ & $8.41 \pm 0.25$ & $8.51 \pm 0.22$ & $8.33 \pm 0.32$ \\
\hline KMD2 & $8.01 \pm 0.52$ & $7.86 \pm 0.40$ & $7.81 \pm 0.48$ & $7.84 \pm 0.50$ & $7.53 \pm 0.46$ & $7.80 \pm 0.66$ \\
\hline KMD3 & $7.52 \pm 0.57$ & $7.22 \pm 0.36$ & $7.22 \pm 0.38$ & $7.06 \pm 0.30$ & $7.00 \pm 0.38$ & $7.36 \pm 0.73$ \\
\hline KMD4 & $6.52 \pm 0.38$ & $6.26 \pm 0.29$ & $6.17 \pm 0.33$ & $5.80 \pm 0.45$ & $5.95 \pm 0.36$ & $6.00 \pm 0.43$ \\
\hline SEM \pm & $\mathbf{0 . 0 9 9 5}$ & $\mathbf{0 . 0 8 5 8}$ & $\mathbf{0 . 0 7 5 8}$ & $\mathbf{0 . 0 8 1 8}$ & $\mathbf{0 . 0 7 6 5}$ & $\mathbf{0 . 1 1 7 3}$ \\
\hline F-value & $* *$ & $* *$ & $* *$ & $* *$ & $* *$ & $* *$ \\
\hline CD at 5\% & $\mathbf{0 . 2 7 9 1}$ & $\mathbf{0 . 2 4 0 8}$ & $\mathbf{0 . 2 1 2 6}$ & $\mathbf{0 . 2 2 9 6}$ & $\mathbf{0 . 2 1 4 6}$ & $\mathbf{0 . 3 2 9 0}$ \\
\hline
\end{tabular}

*significant at $\mathrm{p}<0.05 \%, * *$ significant at $\mathrm{p}<0.01 \%$ 
Table.3 Nutritive value of kodo millet dosa Mix per 100g

\begin{tabular}{|c|c|c|c|c|c|c|c|c|c|}
\hline Variations & $\begin{array}{c}\text { Moisture } \\
(\%)\end{array}$ & $\begin{array}{c}\text { Carbohydrate } \\
\text { (g) }\end{array}$ & $\begin{array}{l}\text { Protein } \\
\text { (g) }\end{array}$ & Fat (g) & Ash (g) & $\begin{array}{c}\text { Crude } \\
\text { fibre (g) }\end{array}$ & $\begin{array}{c}\text { Calcium } \\
\text { (mg) }\end{array}$ & Iron(mg) & $\begin{array}{c}\text { Energy } \\
\text { K.cal }\end{array}$ \\
\hline CMD & $11.32 \pm 0.01$ & $57.05 \pm 1.08$ & $12.22 \pm 0.04$ & $0.43 \pm 0.02$ & $2.83 \pm 0.03$ & $\begin{array}{c}15.40 \pm \\
0.25\end{array}$ & $\begin{array}{c}48.08 \pm \\
0.08\end{array}$ & $1.80 \pm 0.02$ & $\begin{array}{c}280.92 \pm \\
4.23\end{array}$ \\
\hline KMD3 & $\begin{array}{c}11.53 \pm \\
0.05\end{array}$ & $55.91 \pm 0.19$ & $11.33 \pm 0.09$ & $0.41 \pm 0.02$ & $3.49 \pm 0.04$ & $\begin{array}{c}17.33 \pm \\
0.05\end{array}$ & $\begin{array}{c}53.83 \pm \\
2.98\end{array}$ & $5.62 \pm 0.64$ & $\begin{array}{c}272.62 \pm \\
0.27\end{array}$ \\
\hline SEM \pm & 0.0191 & 0.1225 & 0.0414 & 0.0103 & 0.0176 & 0.1058 & 1.2169 & 0.2617 & 0.4206 \\
\hline F -value & $* *$ & $* *$ & $* *$ & NS & $* *$ & $* *$ & $*$ & $* *$ & $* *$ \\
\hline CD at $5 \%$ & 0.0752 & 0.4811 & 0.1624 & 0.0403 & 0.0693 & 0.4154 & 4.7780 & 1.0276 & 1.6516 \\
\hline
\end{tabular}

*significant at $\mathrm{p}<0.05 \%$, ** significant at $\mathrm{p}<0.01 \%$ NS-Non Significant

Table.4 Antioxidants, polyphenols, tannins and phytic acid contents in kodo millet dosa mix

\begin{tabular}{|l|c|c|c|c|}
\hline Variations & Total antioxidants $\mathbf{( \% )}$ & Polyphenols $(\mathbf{m g} / \mathbf{1 0 0 g})$ & Tannins (\%) & Phytic acid (g/100g) \\
\hline CMD & $17.89 \pm 0.40$ & $20.58 \pm 0.02$ & $0.84 \pm 0.04$ & $0.57 \pm 0.02$ \\
\hline KMD3 & $30.41 \pm 0.80$ & $23.97 \pm 0.46$ & $1.77 \pm 0.19$ & $0.85 \pm 0.04$ \\
\hline SEM \pm & $\mathbf{0 . 3 6 5 1}$ & $\mathbf{0 . 1 8 8 1}$ & $\mathbf{0 . 0 7 7 0}$ & $\mathbf{0 . 0 1 9 7}$ \\
\hline F - value & $* *$ & $* *$ & $* *$ & $* *$ \\
\hline CD at 5\% & $\mathbf{1 . 4 3 3 6}$ & $\mathbf{0 . 7 3 8 6}$ & $\mathbf{0 . 3 0 2 2}$ & $\mathbf{0 . 0 7 7 4}$ \\
\hline
\end{tabular}

*significant at $\mathrm{p}<0.05 \%, * *$ significant at $\mathrm{p}<0.01 \%$

Table.5 Dosa Mix Free Fatty Acid (\%) on storage

\begin{tabular}{|c|c|c|c|c|c|c|}
\hline Variations & Days & Silver pouch (\%) & Plastic pouch (\%) & SEM \pm & F-value & CD 5\% \\
\hline \multirow[t]{7}{*}{ CDM } & Initial day & $0.28 \pm 0.01$ & $0.29 \pm 0.01$ & 0.0033 & NS & 0.0131 \\
\hline & $30^{\text {th }}$ day & $0.32 \pm 0.03$ & $0.36 \pm 0.03$ & 0.0156 & NS & 0.0614 \\
\hline & $60^{\text {th }}$ day & $0.38 \pm 0.04$ & $0.40 \pm 0.01$ & 0.0176 & NS & 0.0693 \\
\hline & $90^{\text {th }}$ day & $0.40 \pm 0.01$ & $0.49 \pm 0.01$ & 0.0047 & $* *$ & 0.0185 \\
\hline & $120^{\text {th }}$ day & $0.44 \pm 0.01$ & $0.51 \pm 0.02$ & 0.0085 & $* *$ & 0.0334 \\
\hline & $150^{\text {th }}$ day & $0.48 \pm 0.03$ & $0.63 \pm 0.05$ & 0.0213 & $* *$ & 0.0838 \\
\hline & $180^{\text {th }}$ day & $0.51 \pm 0.02$ & $0.77 \pm 0.06$ & 0.0254 & $* *$ & 0.0997 \\
\hline \multicolumn{2}{|l|}{ SEM \pm} & 0.0127 & 0.0185 & & & \\
\hline \multicolumn{2}{|l|}{ F -value } & ** & ** & & & \\
\hline \multicolumn{2}{|l|}{ CD at $5 \%$} & 0.0386 & 0.0560 & & & \\
\hline \multirow[t]{7}{*}{ KMD 3} & Initial day & $0.26 \pm 0.02$ & $0.27 \pm 0.02$ & 0.0094 & NS & 0.0370 \\
\hline & $30^{\text {th }}$ day & $0.37 \pm 0.03$ & $0.43 \pm 0.07$ & 0.0287 & NS & 0.1126 \\
\hline & $60^{\text {th }}$ day & $0.40 \pm 0.02$ & $0.50 \pm 0.08$ & $\mathbf{0 . 0 3 3 0}$ & NS & 0.1296 \\
\hline & $90^{\text {th }}$ day & $0.44 \pm 0.05$ & $0.54 \pm 0.02$ & 0.0194 & $*$ & 0.0763 \\
\hline & $120^{\text {th }}$ day & $0.47 \pm 0.03$ & $0.63 \pm 0.05$ & 0.0233 & $* *$ & 0.0916 \\
\hline & $150^{\text {th }}$ day & $0.51 \pm 0.04$ & $0.77 \pm 0.02$ & 0.0156 & $* *$ & 0.0614 \\
\hline & $180^{\text {th }}$ day & $0.75 \pm 0.06$ & $0.88 \pm 0.03$ & 0.0277 & $*$ & 0.1087 \\
\hline \multicolumn{2}{|l|}{ SEM \pm} & 0.0205 & 0.0265 & & & \\
\hline \multicolumn{2}{|l|}{ F -value } & $* *$ & $* *$ & & & \\
\hline \multicolumn{2}{|l|}{ CD at $5 \%$} & 0.0622 & 0.0804 & & & \\
\hline
\end{tabular}


Table.6 Peroxide value $\left(\mathrm{Meq} \mathrm{O}_{2} / \mathrm{kg}\right)$ on storage

\begin{tabular}{|c|c|c|c|c|c|c|}
\hline Variations & Days & Silver pouch (\%) & Plastic pouch (\%) & SEM \pm & F-value & CD at $5 \%$ \\
\hline \multirow[t]{7}{*}{ CDM } & Initial & $0.97 \pm 0.02$ & $0.94 \pm 0.02$ & 0.0111 & NS & 0.0434 \\
\hline & $30^{\text {th }}$ & $1.95 \pm 0.05$ & $1.91 \pm 0.01$ & 0.0215 & NS & 0.0843 \\
\hline & $60^{\text {th }}$ & $3.09 \pm 0.09$ & $3.15 \pm 0.03$ & 0.0389 & NS & 0.1529 \\
\hline & 90 & $4.25 \pm 0.12$ & $4.16 \pm 0.04$ & 0.0525 & NS & 0.2061 \\
\hline & 120 & $5.47 \pm 0.07$ & $5.50 \pm 0.04$ & 0.0309 & NS & 0.1214 \\
\hline & 150 & $6.38 \pm 0.20$ & $6.31 \pm 0.15$ & 0.1009 & NS & 0.3960 \\
\hline & 180 & $7.16 \pm 0.18$ & $7.36 \pm 0.06$ & 0.0774 & NS & 0.3040 \\
\hline \multicolumn{2}{|l|}{ SEM \pm} & 0.0694 & 0.0439 & & & \\
\hline \multicolumn{2}{|l|}{ F -value } & $* *$ & $* *$ & & & \\
\hline \multicolumn{2}{|l|}{ CD at $5 \%$} & 0.2105 & 0.1332 & & & \\
\hline \multirow[t]{7}{*}{ KMD 3} & Initial & $0.57 \pm 0.09$ & $0.58 \pm 0.02$ & 0.0380 & NS & 0.1492 \\
\hline & $30^{\text {th }}$ & $2.07 \pm 0.13$ & $2.20 \pm 0.13$ & $\mathbf{0 . 0 7 3 3}$ & NS & 0.2879 \\
\hline & $60^{\text {th }}$ & $3.19 \pm 0.02$ & $3.27 \pm 0.08$ & 0.0317 & NS & 0.1245 \\
\hline & 90 & $4.41 \pm 0.20$ & $4.33 \pm 0.29$ & 0.1454 & NS & 0.5707 \\
\hline & 120 & $5.76 \pm 0.11$ & $5.80 \pm 0.17$ & 0.0835 & NS & 0.3279 \\
\hline & 150 & $6.18 \pm 0.24$ & $6.67 \pm 0.29$ & 0.1519 & NS & 0.5963 \\
\hline & 180 & $6.65 \pm 0.30$ & $7.07 \pm 0.07$ & 0.1261 & NS & 0.4952 \\
\hline \multicolumn{2}{|l|}{ SEM \pm} & 0.1038 & 0.1031 & & & \\
\hline \multicolumn{2}{|l|}{ F -value } & $* *$ & $* *$ & & & \\
\hline \multicolumn{2}{|l|}{ CD at $5 \%$} & 0.3148 & 0.3126 & & & \\
\hline
\end{tabular}

$*$ significant at $\mathrm{p}<0.05 \%, * *$ significant at $\mathrm{p}<0.01 \%$, NS-Non Significant

Table.7 Moisture (\%) of dosa mix on storage

\begin{tabular}{|c|c|c|c|c|c|c|}
\hline Variations & Days & Silver pouch (\%) & Plastic pouch (\%) & SEM & F-value & CD \\
\hline \multirow[t]{7}{*}{ CDM } & Initial & $11.33 \pm 0.05$ & $11.30 \pm 0.09$ & 0.0414 & NS & 0.1624 \\
\hline & 30 & $11.35 \pm 0.03$ & $11.46 \pm 0.09$ & 0.0372 & NS & 0.1460 \\
\hline & 60 & $11.44 \pm 0.03$ & $11.47 \pm 0.06$ & 0.0262 & NS & 0.1031 \\
\hline & 90 & $11.51 \pm 0.03$ & $11.56 \pm 0.03$ & 0.0160 & NS & 0.0628 \\
\hline & 120 & $11.80 \pm 0.01$ & $11.74 \pm 0.06$ & 0.0258 & NS & 0.1014 \\
\hline & 150 & $11.88 \pm 0.18$ & $11.97 \pm 0.01$ & 0.0732 & NS & 0.2873 \\
\hline & 180 & $12.45 \pm 0.18$ & $12.66 \pm 0.03$ & 0.0739 & NS & 0.2902 \\
\hline \multicolumn{2}{|c|}{ SEM } & 0.0571 & $\mathbf{0 . 0 3 3 2}$ & & & \\
\hline \multicolumn{2}{|c|}{ F -value } & $* *$ & $* *$ & & & \\
\hline \multicolumn{2}{|l|}{ CD } & 0.1731 & 0.1006 & & & \\
\hline \multirow[t]{7}{*}{ KMD 3} & Initial & $11.53 \pm 0.05$ & $11.51 \pm 0.03$ & 0.0233 & NS & 0.0916 \\
\hline & 30 & $11.62 \pm 0.06$ & $11.66 \pm 0.01$ & 0.0240 & NS & 0.0944 \\
\hline & 60 & $11.73 \pm 0.07$ & $11.77 \pm 0.04$ & $\mathbf{0 . 0 3 3 7}$ & NS & 0.1325 \\
\hline & 90 & $11.81 \pm 0.02$ & $11.84 \pm 0.06$ & 0.0262 & NS & 0.1031 \\
\hline & 120 & $11.92 \pm 0.07$ & $11.98 \pm 0.01$ & 0.0276 & NS & 0.1083 \\
\hline & 150 & $12.20 \pm 0.08$ & $12.19 \pm 0.08$ & 0.0447 & NS & 0.1756 \\
\hline & 180 & $12.57 \pm 0.10$ & $12.51 \pm 0.08$ & 0.0516 & NS & 0.2026 \\
\hline \multicolumn{2}{|l|}{ SEM } & 0.0424 & 0.0300 & & & \\
\hline \multicolumn{2}{|l|}{ F -value } & $* *$ & $* *$ & & & \\
\hline \multicolumn{2}{|l|}{ CD } & 0.1285 & 0.0910 & & & \\
\hline
\end{tabular}

*significant at $\mathrm{p}<0.05 \%, * *$ significant at $\mathrm{p}<0.01 \%$, NS-Non Significant 
Table.8 Organoleptic scores of dosa mix on storage

\begin{tabular}{|c|c|c|c|c|c|c|c|c|}
\hline \multirow{2}{*}{$\begin{array}{l}\text { Storage } \\
\text { Material }\end{array}$} & \multirow[t]{2}{*}{ Variations } & \multirow[t]{2}{*}{ Duration } & \multicolumn{6}{|c|}{ Sensory attributes } \\
\hline & & & Appearance & Colour & Texture & Aroma & Taste & $\begin{array}{l}\text { Overall } \\
\text { acceptability }\end{array}$ \\
\hline \multirow{20}{*}{$\begin{array}{l}\text { Silver } \\
\text { pouch }\end{array}$} & \multirow{10}{*}{ CDM } & Initial & $8.57 \pm 0.37$ & $8.55 \pm 0.50$ & $8.57 \pm 0.20$ & $8.60 \pm 0.27$ & $8.60 \pm 0.23$ & $8.34 \pm 0.36$ \\
\hline & & $30^{\text {th }}$ day & $8.44 \pm 0.38$ & $8.36 \pm 0.66$ & $8.47 \pm 0.28$ & $8.54 \pm 0.32$ & $8.45 \pm 0.53$ & $8.24 \pm 0.54$ \\
\hline & & $60^{\text {th }}$ day & $8.39 \pm 0.66$ & $8.31 \pm 0.85$ & $8.35 \pm 0.59$ & $8.49 \pm 0.36$ & $8.40 \pm 0.31$ & $8.20 \pm 0.51$ \\
\hline & & $90^{\text {th }}$ day & $8.34 \pm 0.72$ & $8.27 \pm 0.77$ & $8.29 \pm 0.67$ & $8.43 \pm 0.36$ & $8.37 \pm 0.37$ & $8.17 \pm 0.55$ \\
\hline & & $120^{\text {th }}$ day & $8.31 \pm 0.65$ & $8.10 \pm 0.83$ & $8.20 \pm 0.72$ & $8.30 \pm 0.70$ & $8.30 \pm 0.61$ & $8.12 \pm 0.65$ \\
\hline & & $150^{\text {th }}$ day & $8.28 \pm 0.59$ & $8.00 \pm 0.89$ & $8.12 \pm 0.70$ & $8.19 \pm 0.69$ & $8.11 \pm 0.74$ & $7.98 \pm 0.70$ \\
\hline & & $180^{\text {th }}$ day & $7.98 \pm 0.86$ & $7.90 \pm 0.94$ & $7.95 \pm 0.80$ & $7.96 \pm 0.88$ & $7.99 \pm 0.82$ & $7.60 \pm 1.19$ \\
\hline & & F value & $* *$ & $* *$ & $* *$ & $* *$ & $* *$ & $* *$ \\
\hline & & SEm \pm & 0.1368 & 0.1724 & 0.1320 & 0.1216 & 0.1212 & 0.1514 \\
\hline & & CD (5\%) & 0.3824 & 0.4819 & 0.3690 & 0.3399 & 0.3388 & 0.4233 \\
\hline & \multirow[t]{10}{*}{ KMD 3} & Initial & $7.52 \pm 0.58$ & $7.22 \pm 0.37$ & $7.22 \pm 0.39$ & $7.06 \pm 0.30$ & $7.00 \pm 0.39$ & $7.36 \pm 0.74$ \\
\hline & & $30^{\text {th }}$ day & $7.44 \pm 0.60$ & $7.06 \pm 0.57$ & $7.18 \pm 0.38$ & $7.04 \pm 0.36$ & $6.99 \pm 0.44$ & $7.26 \pm 0.90$ \\
\hline & & $60^{\text {th }}$ day & $7.37 \pm 0.55$ & $7.02 \pm 0.56$ & $7.13 \pm 0.38$ & $7.00 \pm 0.25$ & $6.94 \pm 0.45$ & $7.21 \pm 0.83$ \\
\hline & & $90^{\text {th }}$ day & $7.35 \pm 0.60$ & $6.96 \pm 0.57$ & $7.05 \pm 0.58$ & $6.95 \pm 0.43$ & $6.88 \pm 0.51$ & $7.17 \pm 0.98$ \\
\hline & & $120^{\text {th }}$ day & $7.30 \pm 0.58$ & $6.79 \pm 0.65$ & $6.98 \pm 0.62$ & $6.43 \pm 0.91$ & $6.80 \pm 0.58$ & $7.10 \pm 0.82$ \\
\hline & & $150^{\text {th }}$ day & $7.05 \pm 0.67$ & $6.71 \pm 0.64$ & $6.88 \pm 0.75$ & $6.43 \pm 0.91$ & $6.64 \pm 0.76$ & $7.00 \pm 0.88$ \\
\hline & & $180^{\text {th }}$ day & $6.33 \pm 1.02$ & $6.67 \pm 0.80$ & $6.76 \pm 0.90$ & $6.33 \pm 0.90$ & $6.58 \pm 0.76$ & $6.95 \pm 0.85$ \\
\hline & & F value & $* *$ & $* *$ & $* *$ & ** & $* *$ & $* *$ \\
\hline & & SEm \pm & 0.1470 & 0.1321 & 0.1311 & 0.1416 & 0.1250 & 0.1874 \\
\hline & & CD (5\%) & 0.4111 & 0.3693 & 0.3665 & 0.3960 & 0.3494 & 0.5239 \\
\hline \multirow{20}{*}{$\begin{array}{l}\text { Plastic } \\
\text { pouch }\end{array}$} & \multirow{10}{*}{ CDM } & Initial & $8.58 \pm 0.38$ & $8.49 \pm 0.53$ & $8.53 \pm 0.23$ & $8.54 \pm 0.32$ & $8.56 \pm 0.26$ & $8.31 \pm 0.37$ \\
\hline & & $30^{\text {th }}$ day & $8.49 \pm 0.39$ & $8.34 \pm 0.68$ & $8.41 \pm 0.30$ & $8.51 \pm 0.34$ & $8.47 \pm 0.44$ & $8.20 \pm 0.51$ \\
\hline & & $60^{\text {th }}$ day & $8.32 \pm 0.65$ & $8.22 \pm 0.82$ & $8.33 \pm 0.60$ & $8.45 \pm 0.37$ & $8.37 \pm 0.33$ & $8.17 \pm 0.51$ \\
\hline & & $90^{\text {th }}$ day & $8.27 \pm 0.71$ & $8.17 \pm 0.86$ & $8.24 \pm 0.67$ & $8.38 \pm 0.47$ & $8.32 \pm 0.36$ & $8.12 \pm 0.59$ \\
\hline & & $120^{\text {th }}$ day & $8.22 \pm 0.82$ & $8.07 \pm 0.84$ & $8.22 \pm 0.69$ & $8.29 \pm 0.66$ & $8.30 \pm 0.61$ & $8.08 \pm 0.69$ \\
\hline & & $150^{\text {th }}$ day & $8.19 \pm 0.70$ & $8.02 \pm 0.90$ & $8.20 \pm 0.66$ & $8.24 \pm 0.64$ & $8.05 \pm 0.72$ & $7.98 \pm 0.70$ \\
\hline & & $180^{\text {th }}$ day & $7.98 \pm 0.86$ & $7.83 \pm 0.89$ & $7.90 \pm 0.82$ & $7.98 \pm 0.84$ & $7.95 \pm 0.80$ & $7.60 \pm 1.19$ \\
\hline & & F value & $* *$ & $* *$ & $* *$ & $* *$ & $* *$ & $* *$ \\
\hline & & SEm \pm & 0.1460 & 0.1740 & 0.1313 & 0.1201 & 0.1172 & 0.1500 \\
\hline & & CD (5\%) & 0.4083 & 0.4865 & 0.3671 & 0.3358 & 0.3277 & 0.4195 \\
\hline & \multirow[t]{10}{*}{ KMD 3} & Initial & $7.48 \pm 0.58$ & $7.20 \pm 0.33$ & $7.24 \pm 0.41$ & $7.04 \pm 0.30$ & $7.03 \pm 0.36$ & $7.36 \pm 0.74$ \\
\hline & & $30^{\text {th }}$ day & $7.39 \pm 0.67$ & $7.03 \pm 0.56$ & $7.22 \pm 0.48$ & $7.05 \pm 0.44$ & $6.98 \pm 0.44$ & $7.29 \pm 0.94$ \\
\hline & & $60^{\text {th }}$ day & $7.34 \pm 0.53$ & $7.02 \pm 0.54$ & $7.19 \pm 0.46$ & $7.02 \pm 0.28$ & $6.96 \pm 0.45$ & $7.24 \pm 0.87$ \\
\hline & & $90^{\text {th }}$ day & $7.28 \pm 0.68$ & $7.00 \pm 0.52$ & $7.14 \pm 0.73$ & $7.01 \pm 0.38$ & $6.92 \pm 0.47$ & $7.21 \pm 1.11$ \\
\hline & & $120^{\text {th }}$ day & $7.25 \pm 0.65$ & $6.81 \pm 0.63$ & $7.00 \pm 0.63$ & $6.90 \pm 0.52$ & $6.89 \pm 0.51$ & $7.19 \pm 0.75$ \\
\hline & & $150^{\text {th }}$ day & $7.10 \pm 0.62$ & $6.76 \pm 0.62$ & $6.95 \pm 0.66$ & $6.62 \pm 0.96$ & $6.63 \pm 0.77$ & $7.02 \pm 0.75$ \\
\hline & & $180^{\text {th }}$ day & $6.33 \pm 1.02$ & $6.69 \pm 0.72$ & $6.78 \pm 0.81$ & $6.48 \pm 0.86$ & $6.75 \pm 0.96$ & $7.00 \pm 0.82$ \\
\hline & & F value & $* *$ & $* *$ & $* *$ & $* *$ & $* *$ & $* *$ \\
\hline & & SEm \pm & 0.1498 & 0.1244 & 0.1340 & 0.1286 & 0.1310 & 0.1938 \\
\hline & & CD (5\%) & 0.4188 & 0.3479 & 0.3745 & 0.3595 & 0.3661 & 0.5419 \\
\hline
\end{tabular}

*significant at $\mathrm{p}<0.05 \%, * *$ significant at $\mathrm{p}<0.01 \%$ 
Table.9 Microbial load of value added dosa mix

\begin{tabular}{|c|c|c|c|c|}
\hline \multirow[t]{2}{*}{ Organisms } & \multirow[t]{2}{*}{ Variations } & \multicolumn{3}{|c|}{ Duration } \\
\hline & & Initial day & $90^{\text {th }}$ day & $180^{\text {th }}$ day \\
\hline \multirow{4}{*}{$\begin{array}{l}\text { Bacteria } \\
\left(\times 10^{2} \mathrm{cfu} / \mathrm{g}\right)\end{array}$} & CMD (SP) & $0.00(0.707)$ & $0.00(0.707)$ & $0.66(1.077)$ \\
\hline & KMD3 (SP) & $0.00(0.707)$ & $0.00(0.707)$ & $0.66(1.077)$ \\
\hline & CMD (PP) & $0.00(0.707)$ & $0.00(0.707)$ & $0.66(1.077)$ \\
\hline & KMD3 (PP) & $0.00(0.707)$ & $0.00(0.707)$ & $0.33(0.911)$ \\
\hline \multirow{4}{*}{$\begin{array}{l}\text { Fungi } \\
\left(\times 10^{2} \mathrm{cfu} / \mathrm{g}\right)\end{array}$} & CMD (SP) & $0.00(0.707)$ & $0.00(0.707)$ & $0.00(0.707)$ \\
\hline & KMD3 (SP) & $0.00(0.707)$ & $0.00(0.707)$ & $0.00(0.707)$ \\
\hline & CMD (PP) & $0.00(0.707)$ & $0.00(0.707)$ & $0.00(0.707)$ \\
\hline & KMD3 (PP) & $0.00(0.707)$ & $0.00(0.707)$ & $0.00(0.707)$ \\
\hline \multirow{4}{*}{$\begin{array}{l}\text { Coliforms } \\
\left(\times 10^{2} \text { cfu/g) }\right.\end{array}$} & CMD (SP) & $0.00(0.707)$ & $0.00(0.707)$ & $0.00(0.707)$ \\
\hline & KMD3 (SP) & $0.00(0.707)$ & $0.00(0.707)$ & $0.00(0.707)$ \\
\hline & CMD (PP) & $0.00(0.707)$ & $0.00(0.707)$ & $0.00(0.707)$ \\
\hline & KMD (PP) & $0.00(0.707)$ & $0.00(0.707)$ & $0.00(0.707)$ \\
\hline
\end{tabular}

CMD (SP)-Control Millet Dosa (silver pouch), KMD(SP)-Kodo Millet Dosa (silver pouch)

CMD (PP)-Control Millet Dosa (plastic pouch), KMD(PP)-Kodo Millet Dosa (plastic pouch), Values in parenthesis indicate $(\sqrt{ } x+0.5)$

Fig.1 Flow chart for preparation of kodo millet dosa mix

Kodo millet grits, parboiled rice, fenugreek seeds and black gram dhal were separately ground to powder.

Then mixed together all the ingredients

Salt was finally added

Fig.2 Flow chart for preparation of kodo millet dosa

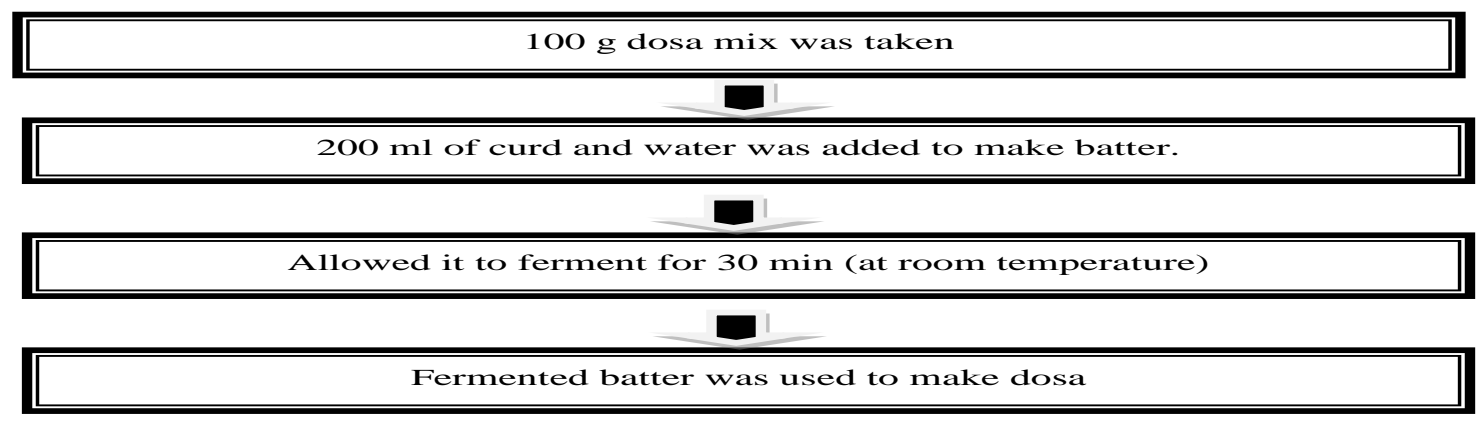


Table 6 indicates the peroxide values of the dosa mix. In control dosa mix, peroxide values when stored in silver pouch increased significantly from 0.97 to 7.16 , in plastic pouch from 0.94 to 7.36 . In kodo dosa mix it was increased from 0.57 to 6.65 in silver pouch, and from 0.58 to 7.07 in plastic pouch. Significant difference was not observed between type of storage materials. Peroxide values were within safe limits upto $180^{\text {th }}$ day of storage period.

Table 7 denotes the moisture content of stored dosa mix. Control dosa mix in silver pouch showed significant increase of 11.33 to 12.45 , in plastic pouch from 11.30 to 12.66 . In kodo dosa mix stored in silver pouch was from 11.53 to 12.57 and in plastic pouch was from 11.51 to 12.51 . This may be due to hygroscopic nature of flours.

Table 8 shows the organoleptic scores of stored products. Control dosa mix stored in silver pouch appearance scores reduced from 8.57 to 7.98 , colour from 8.55 to 7.90 , texture 8.57 to 7.95 , aroma 8.60 to 7.96 , taste 8.60 to 7.99 and overall acceptability from 8.34 to 7.60. KMK6 scores got reduced for appearance from 7.52 to 6.33 , for colour 7.22 to 6.67 , texture 7.22 to 6.76 , aroma 7.06 to 6.33 , taste 7.00 to 6.58 and overall acceptability 7.36 to 6.95 .

In plastic pouch also, in control dosa mix scores got reduced for appearance from 8.58 to 7.98 , for colour 8.49 to 7.83 , texture 8.53 to 7.90 , aroma 8.54 to 7.98 , taste 8.56 to 7.95 and overall acceptability 8.31 to 7.60 . For kodo dosa mix scores reduced for appearance from 7.48 to 6.33 , for colour 7.20 to 6.69 , texture 7.24 to 6.78 , aroma 7.04 to 6.48 , taste 7.03 to 6.75 and overall acceptability 7.36 to 7.00 .

Thus, on $180^{\text {th }}$ day, the overall acceptability of kodo dosa mix was 6.95 and 7.00 stored in silver and plastic pouches respectively, indicating the moderate level of acceptability.

Table 9 denotes the microbial load of dosa mix. There was no growth of any kind of microorganisms on initial to $90^{\text {th }}$ day of storage both in silver pouch and plastic pouch packed products. But bacteria started to grow on $180^{\text {th }}$ day in products i.e. both control and kodo dosa mixes packed in silver as well as plastic pouches. However, Fungi and coliforms were not observed. Moreover, bacterial load was within permissible safe limits. Thus, the results showed that dosa mix can be safely stored for 180 days at ambient temperature either in plastic or silver pouches.

In conclusion the millets are climate resilient and nutritionally superior crops. Hence, there is a need to develop value added products from millets to exploit their nutritional and health benefits. From the present study, it is observed that traditional ready to prepare, acceptable and shelf stable instant dosa mix can be successfully developed. However, such products should be popularized among all sectors of population.

\section{Acknowledgement}

The authors gratefully acknowledge the World Bank funded NAHEP-CAAST Project on NGT_AA operating at UAS, Bengaluru for providing the financial support.

\section{References}

A.O.A.C. (1980). Official methods of analysis 13th Edition. Association of Official analytical chemists, Washington, D.C.

A.O.A.C. (2000). Official Methods of Analysis, 17th Ed., Association of Official analytical chemists, Washington D.C.

E.L.Wheeler and R.E. Ferrel (1971). A method for phytic acid determination 
in wheat and wheat fractions. Agricultural research service, U.S. department of Agriculture, California.

Heau, W.R., Menzel, R.G., Roberts, H. and Freee, M. H. (1965) Methods of soil and plant analysis. Agriculture research service, Department of Agriculture, USA.

Kang, H.M. and Saltveit, M.E. (2002) Antioxidant capacity of lettuce leaf tissue increases after wounding. $J$. Agric. Food .Chem., 50:7536-7541.

Livesey, G. (1995) Metabolizable energy of macro nutrients. Am. J. Clin. Nutr.,62 : $1135-1142$.

Mounika M. Uma Devi K., and Sucharitha Devi S. (2017). Iron Bioavailability from Little Millet and Proso Millet Based Recipes. Int.J.Curr.Microbiol. App.Sci., 6(10): 2832-2840.

Muragod, P. P, Muruli, N.V., Padeppagol S. and Kattimani A. (2019). Develop the Value Added Products and Evaluate the Storage Quality of Kodo Millet Grains Products. Int. J. Pure App. Biosci. 7 (1): 97-107.

Perez-Jimenez J, Neveu V, Vos F, Scalber A. (2010), Identification of the 100 richest dietary sources of polyphenols: an application of the Phenol-Explorer database. Eur J Clin Nutr. 64:S11220.
Ranganna, S. (2005). Hand book of analysis and quality control of fruit and vegetable products, Tata McGraw Hill. Third edition: 868-925.

Roopa S. S., Dwivedi, H. and Rana, G.K. (2017). Development and physical, nutritional and sensory evaluation of instant mix (DOSA). TECHNOFAMEA Journal of Multidisciplinary Advance Research. Vol.6 No. 1, 109113.

Sadasivam, S. and Manickam, A. (1991) Biochemical methods, second edition. New age Int Publishers, Coimbatore.

Shreshta, A K. and Noomhorm, A. (2002). Comparison of physico-chemical properties of biscuits supplemented with soy and Kinema flours. International Journal of Food Science and Technology. 37: 361-368.

Singh A, Holvoet S, Mercenier A. (2011), Dietary polyphenols in the prevention and treatment of allergic diseases. Clin Exp Allergy, 41:1346-59.

Vidhya A.M., and Roobhadevi M. (2014). Formulation and Shelf life Evaluation of Kodo Millet Incorporated Ready to Cook Mixes. Indian Journal of Research in Food Science and Nutrition, Vol 1(1), 27-31.

\section{How to cite this article:}

Vijayalaxmi, K. G., Jayalaxmi Baddi and Vanishri K. Umarji. 2020. A Study on Development of Instant Kodo Dosa Mix and Evaluation of Its Nutritional Composition and Shelf Life. Int.J.Curr.Microbiol.App.Sci. 9(12): 286-295. doi: https://doi.org/10.20546/ijcmas.2020.912.038 